\title{
Simulation of Event-Based Technique for Harmonic Failures
}

\author{
Futra Zamsyah Md Fadzil ${ }^{1}$ Ali Mousavi ${ }^{2}$ and Morad Danishvar ${ }^{3}$
}

\begin{abstract}
An event-based technique to determine the optimum performance of power plants is proposed. Also known as Event Modeller, the technique is borrowed from the EventTracker principle [1] and Event Clustering method [2], to build on cause-effect relationships of events that potentially link many system inputs to many system outputs. Various signal processing methods use Feature Extraction, Feature Selection and Classifier to detect, solve, and mitigate Power Quality (PQ) Disturbances problems. However, most of the solutions are limited to its internal system parameters and require high computational effort. The Event Modeller, on the other hand, considers both internal and external or environmental parameters, which have the potential to fill the gap to overcome limitations of the $P Q$ problems. The algorithm is very selective as it only focuses on the uniqueness of the real-time data, making it an alternative classification technique to detect the PQ problem. A real-time simulation of a Continuous Ship Unloader (CSU) machine is presented to demonstrate the applicability of the Event Modeller in detecting the harmonic failure which considers the dynamics and the interactions within the plant machinery.
\end{abstract}

\section{INTRODUCTION}

Power Quality (PQ) monitoring has been the focus of research and development for many years. The main focus is to protect the equipment and minimise the losses while increasing the levels of operational safety. However, systems are becoming more complex as they are developed. Huge numbers of control drives and other non-linear loads have been installed to meet the demands of modern lifestyles. It has led to power system instability, thus creating high noises in the system grids and decayed the electrical distribution system. Even worse, climate change has an impact on the environment that the system operates in. In some cases especially in hot countries, the electrical distribution system requires an air conditioner to protect the electrical devices in the substation. In normal circumstances, if the air conditioner is a malfunction, it may exceed the allowable set point and fail to protect the equipment. When the global temperature increases, the probability rate of failure also increases. Research has found that the long-term average global temperature is rising which have a significant negative impact on the performance of the machines [3].

Earlier, the conventional methods use manual configurations and visual inspection to monitor the quality of the

${ }^{1}$ Futra Zamsyah Md Fadzil is with Electronic \& Computer Engineering, Brunel University, Kingston Lane, UB8 3PH, Uxbridge, United Kingdom. FutraZamsyah.MdFadzilebrunel.ac.uk

${ }^{2}$ Ali Mousavi is with Electronic \& Computer Engineering, Brunel University, Kingston Lane, UB8 3PH, Uxbridge, United Kingdom. Ali.Mousavi@brunel.ac.uk

${ }^{3}$ Morad Danishvar is with Electronic \& Computer Engineering, Brunel University, Kingston Lane, UB8 3PH, Uxbridge, United Kingdom. Morad.Danishvarabrunel.ac.uk power supply in a system. This method was too difficult to interpret and time-consuming. Later, the automatic classification method, which uses the signal processing technique was introduced and currently progressing with various artificial intelligence learning techniques. Different combination of feature extraction and classifiers have proven to locate the PQ disturbance accurately using synthetic data. However, in real industry data, the results may vary. The real industry data are complex, and it is even more complicated when there are external or environmental parameters, which could potentially influence the system state. Thus, it creates a gap in the existing techniques, which motivate the authors to explore the relevance of this environmental data towards PQ disturbance problems. Besides that, as the modern data are even more complex, system engineer has to ensure that the system is capable of running the learning techniques in realtime application. Disturbance data occurs in the order of microseconds, which increases the record data significantly [4]. Thus, it will burden the learning classifiers to run unnecessary parameters that result in high computation. Therefore, the authors are suggesting a method that could fill the gap, by taking into account the external parameters in the system state. This technique also capable of removing all the logical boundaries of isolation, that exists in the complex system, while filtering the unwanted data. Moreover, the Event Modeller system is capable of including the information that was thought irrelevant at the outset [5].

This paper aims to introduce an event-based analysis as a technique to detect harmonics failure in real-time. The technique is capable of grouping the high correlation system parameters together, to form an input-output relationship, which is not limited to internal parameters only. As such, a homogenised correlation system will suggest the possible root cause of the harmonic failures, while eliminating the unimportant parameters in real-time.

In this paper, a real-time simulation of CSU machines is presented, which demonstrates the changes to output parameters on input activity. This data will be used to measure the suitability and applicability of the event modeller techniques in the power system environment.

\section{A. Power Quality Disturbance}

The PQ disturbances are mainly due to the following reasons [6]:

1) the increase in numbers of complex power electronic devices, variable speed drives, power factor corrections in the industry sector.

2) the rapid increase in the domestic, commercial and industrial use of computers. 


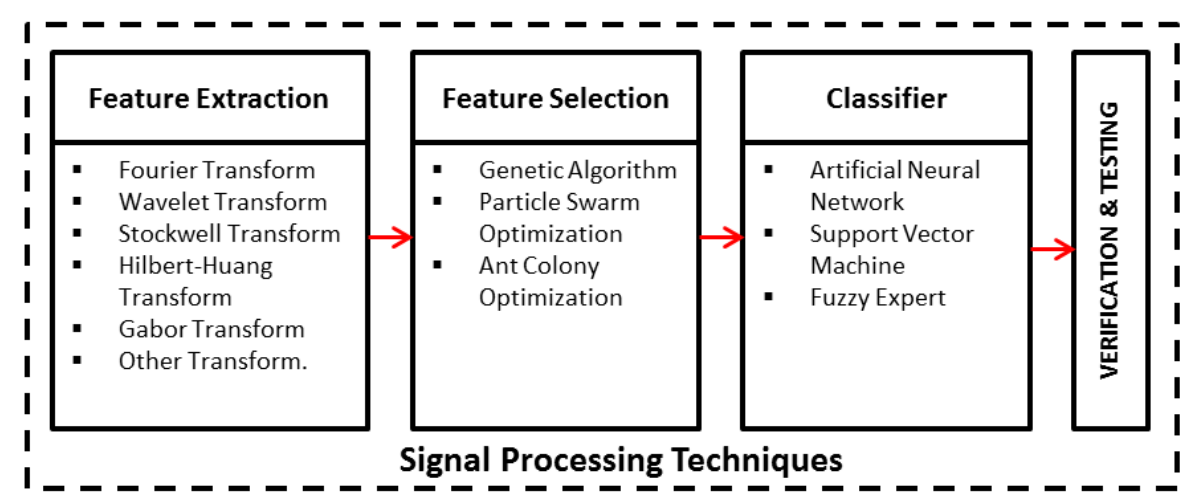

Fig. 1. Various techniques of Power Quality Classification

3) the increased number of renewable energy plants.

4) the complexity of industrial processes and interconnections of systems.

5) the new design of better-performance equipment which adds new sources of PQ disturbances in the system

There are a number of different types of PQ disturbances which include Voltage Sag, Voltage Swell, Transients, Harmonics, Fluctuations, Flicker, Voltage Unbalance, Interruption, DC Offset and Notches. An efficient way to diagnose these PQ disturbances requires a high level of engineering expertise [7].

The PQ disturbance mitigation is required to avoid power disruption between the power utility and the end user. With modern technology, much research has been devoted to mitigating this problem using the signal processing method. This method has three principal stages which incorporate feature extraction, feature selection and classification.

Feature extraction is a technique to extract useful information from a hidden PQ signals/waveform. It may directly be extracted from the original measurement either from some transformed domain or from the parameters of signal models [8]. Several approaches includes short-time Fourier transform [9], wavelet transform [10], wavelet packet transform [11], Hilbert Huang transform [12], Stockwell transform [13], Gabor-Wigner transform [14], and other hybrid transform based [15].

Feature selection is a process of selecting the most useful and relevant data to ensure low redundancy in classifying the PQ Disturbance. Based on the extraction data, optimisation techniques such as Genetic Algorithm [16], Particle Swarm Optimisation [17] and Ant Colony Optimisation [18] are the common techniques used in PQ disturbance.

Classification is a process of predicting the class of given data points. It uses an algorithm to implements classification by approximating a mapping function from the input variable to output variables. Artificial Neural Network is among the most popular and influential classes of machine learning algorithms. Some other machine learning includes Support Vector Machine [19] and fuzzy expert system [20].

Figure 1 above shows the arrangement of the three principal stages in PQ classification. A detailed review of these techniques can be found in [21], [22] and [23].

\section{B. Power Quality Standards}

Harmonic evaluations of the utility systems involve procedures to make sure that the quality of the voltage supplied to all customers is acceptable [24]. However, most of the harmonic problems occur at the consumer end. Their devices contain non-linear loads, which result in resonance conditions [24]. These non-linear loads are the current sources of harmonics. The system voltage appears stiff to individual loads, and the loads draw distorted current waveforms. Therefore, there is a need to maintain a PQ International standard, to provide the guidelines and limits for the acceptable levels of compatibility, between consumer equipment and the system utilities.

The International Standard IEEE 519-1992 sets the limit for both harmonic voltages and currents at the Point of Common Coupling (PCC) between the end user and the utility supplier. It limits Voltage Total Harmonic Distortion (THD), defined as the ratio of the RMS value of the harmonic voltage to the RMS value of the fundamental $(50 \mathrm{~Hz})$ voltage, to a maximum of $5 \%$. Individual voltage harmonic magnitudes are limited to $3 \%$ of the fundamental voltage value [25].

The International Standard IEC 61000-3-4 sets the limits for emission of harmonic currents in low-voltage power supply systems for the requirement with rated current greater than 16A [26].

\section{The Environmental Parameters}

It is the regulation for both utilities company and consumers to comply with the International PQ Standards. To sustain a good power quality system, a tremendous amount of research and PQ monitoring has been done to classify these PQ disturbance problems. However, there is still a shortfall in solving the PQ problem effectively. Most of the solutions are limited to the signal processing parameters within the system. The solution does not consider the external parameters which could have a significant impact on the problem. [27] suggested that some machines fail due to the operations activity and/or environmental factors. The gap motivates the authors to further investigate the relevance of the external parameters to the PQ problems. Therefore, the authors are proposing 


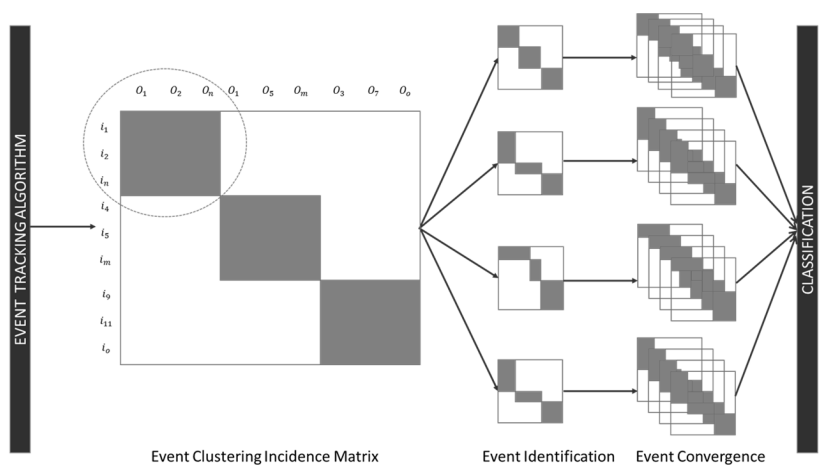

Fig. 2. Event Modeller Techniques

the Event Modeller techniques which could link the internal and external parameters together, to create a cause-effect relationship, while updating the system status in near realtime before the machine fails. The contribution of this technique will be weight based on the application of a novel real-time learning technique. The learning technique will automatically reformulate the possible relationships between the internal and external parameters. Thus, it specifies the PQ parameter out of other control and machine parameters which could lead to PQ failure. The further information on Event Modeller technique are described as follows:

\section{Event Modeller Technique}

The event modeller technique is designed to evaluate the relationship between the actual events (Output Data) to the cause of the triggered events (Input Data) by using a data mapping concept. It groups the high correlation system parameters in the form of matrices and places them into mutually exclusive blocks. This form an input-output relationship, which take into account both internal and external factors. The blocks are sorted based on the events coincidence and events similarity. Each block represents a profile of a similar event scenario, which then trained in the network to converge the same entities together for classification purposes. One of the important aspects of this Event Modeller technique is its potential to include information that was initially thought irrelevant or not considered at the design stage. Figure 2 illustrates the event modeller techniques which comprises of Event Tracking, Event Clustering, Event Identification, Event Convergence and Event Classification.

\section{A. EventTracker Algorithm}

The event-based sensitivity analysis method or also known as EventTracker [28] is an input variable selection that correlates between system input and system output, to construct a discrete event framework [29]. The events are loosely coupled, with respect to their triggers for the purpose of sensitivity analysis. This method supports the time-critical dimensionality reduction problem with limited computational resources, which make it suitable for feature extraction. The key objective of this algorithm is to record all events, while eliminating the non-important data which is not sensitive
TABLE I

BASIC DESCRIPTION OF EVENT-MODELLER

\begin{tabular}{|c|c|c|}
\hline Description & Definition & Impact \\
\hline $\begin{array}{l}\text { Discrete } \\
\text { Event } \\
\text { System }\end{array}$ & $\begin{array}{l}\text { The disparate } \\
\text { occurrence of } \\
\text { events in a } \\
\text { specified time span. }\end{array}$ & $\begin{array}{l}\text { Any changes in the } \\
\text { input/output will } \\
\text { change the system } \\
\text { state. }\end{array}$ \\
\hline $\begin{array}{l}\text { Triggered } \\
\text { Data } \\
\text { (TD) }\end{array}$ & $\begin{array}{l}\text { Any input variable } \\
\text { whose value } \\
\text { transition registered } \\
\text { an event. }\end{array}$ & $\begin{array}{l}\text { Any Individual or } \\
\text { combination of input } \\
\text { variables may have } \\
\text { different effects on } \\
\text { different system outputs } \\
\mathrm{ED}=\{\mathrm{TD} 1, \mathrm{TD} 2,, \mathrm{TDn}\}\end{array}$ \\
\hline $\begin{array}{l}\text { Event } \\
\text { Data } \\
\text { (ED) }\end{array}$ & $\begin{array}{l}\text { The series of data } \\
\text { that represent the } \\
\text { state of the system } \\
\text { at a given time }\end{array}$ & $\begin{array}{l}\text { Any Individual or } \\
\text { combination of input } \\
\text { variables may have } \\
\text { different effects on } \\
\text { different system outputs } \\
\mathrm{ED}=\{\mathrm{TD} 1, \mathrm{TD} 2,, \mathrm{TDn}\}\end{array}$ \\
\hline $\begin{array}{l}\text { Triggered } \\
\text { Threshold } \\
\text { (TT) }\end{array}$ & $\begin{array}{l}\text { A given numerical } \\
\text { value set point that } \\
\text { the values of TD } \\
\text { series based on } \\
\text { experts/ } \\
\text { historical data. }\end{array}$ & $\begin{array}{l}\text { The fluctuations in the } \\
\text { TD series that are } \\
\text { interpreted as triggers } \\
\text { are determined in } \\
\text { comparison with } \\
\text { the TT. }\end{array}$ \\
\hline $\begin{array}{l}\text { Event } \\
\text { Threshold } \\
\text { (ET) }\end{array}$ & $\begin{array}{l}\text { A proportion or } \\
\text { percentage of an } \\
\text { overall range of } \\
\text { values of TD series } \\
\text { over the time scale. }\end{array}$ & $\begin{array}{l}\text { The fluctuations in the } \\
\text { ED series that are } \\
\text { interpreted as triggers } \\
\text { are determined in } \\
\text { comparison with the ET. }\end{array}$ \\
\hline $\begin{array}{l}\text { Search } \\
\text { Slot } \\
(\mathrm{SS})\end{array}$ & $\begin{array}{l}\text { A fixed time slot } \\
\text { within which } \\
\text { batches of TD and } \\
\text { ED are captured. }\end{array}$ & $\begin{array}{l}\text { The fixed time slot } \\
\text { (scan rate) is } \\
\text { determined by experts. }\end{array}$ \\
\hline $\begin{array}{l}\text { Analysis } \\
\text { Span } \\
\text { (AS) }\end{array}$ & $\begin{array}{l}\text { The time span } \\
\text { within which a } \\
\text { period of } \\
\text { sensitivity } \\
\text { analysis occurs. }\end{array}$ & $\begin{array}{l}\text { Comprised of a } \\
\text { number of } \\
\text { consecutive SS. }\end{array}$ \\
\hline
\end{tabular}

to the Triggered Data (TD). Then, it is translated into a sensitivity index before it is added from the subsequent search and linearly normalised. Based on a number of iterations, it will verify known relations and maps new unknown relationships. A detailed description of the EvenTracker algorithm including the primary information of the Discrete Event System (DES), Trigger Data (TD), and Event Data (ED) is shown in Table I.

\section{B. Event Clustering Algorithm}

The Event Clustering Algorithm (EventiC) [5] is the extension of the EventTracker algorithm, aiming to create a logical interrelationships of the components, and its dynamics within the embedded system in real-time applications. It could reveal the causal relationships that exist between a system and its operational environment. It is designed based on Event-Driven Incidence Matrices (EDIM), which sorts the input (rows) and output (columns) parameter.

The main difference between EventTracker and EventiC resides in the number of outputs. EventTracker analyses multiple inputs with a single output relationship while the EventiC analyse multiple inputs and multiple output relationship. This relationship has made the EventiC a unique tool, as it does not rely on any prejudgement of data relevancy. It also 
has the capability of identifying new influential parameters that were previously unknown [2]. The interpreting rate is fast which only focuses on the unique coincidence activity between the input and output. With that, it reduces the computational cost, which made it relevant to operate in near real-time for a large data processing scale.

The algorithm uses Rank Order Clustering (ROC), which was initially introduced by King, (1980) [30] by rearranging the row and column of a matrix in the iterative manner of decreasing value order. The algorithm calculates the relationship and produces a weight for further analysis. A detailed explanation of EventiC algorithm, which includes a definition of a Key Performance Indicator (KPI) and the basic concept and parameters are discussed in [5].

For optimisation purposes, the authors have improved the existing EventiC algorithm. The following are the step by step implementation of the new event clustering.

1) Set Event Modeller Limit (for e.g. EML = 0.8)

2) Set Threshold Setting (for e.g. Th $=0.05$ )

3) Set Triggered Data as Analogue or Boolean

a) Populate the ULTh and LLTh

4) Populate All Input Data (TD1, TD2...TDn)

a) If Triggered Data $=$ Analogue,

Compute TDx = TDn - TDn-1;

Compare TDx with ULTh and LLTh;

i) If (LLTh $<$ TDx $<$ ULTh); $\mathrm{TDx}=0$;

ii) Else $\mathrm{TDx}=1$.

b) Else Triggered Data $=$ Boolean. $\mathrm{TDx}=\mathrm{TD} 1, \mathrm{TD} 2 \ldots \mathrm{TDn}$.

5) Populate All Output Data (ED1,ED2...EDn);

a) Compute EDx = EDn - EDn-1;

Compare EDx with ULTh and LLTh;

i) If (LLTh $<\mathrm{EDx}<\mathrm{ULTh})$;

$\mathrm{EDx}=0$;

ii) Else $\mathrm{EDx}=1$

6) Populate the models input-output event coincidence matrix with binary weighting values of exclusive NOR function.

7) Average each input-output event coincidence.

8) Sort rows of the resultant binary matrix into decreasing order of their decimal weights.

9) Repeat steps 5 to 7 for every column.

10) Repeat steps 5 to 8 until the position of each element in each row and column does not change.

11) A weight for each row $i$ and column $j$ (in a $m$ by $n$ matrix) is calculated using equation below:

$$
\begin{aligned}
& \text { Row1 : } W_{i}=\sum_{K=1}^{n} a_{i k} 2^{n-k} \\
& \text { Row } 2: W_{i}=\sum_{K=1}^{m} a_{k j} 2^{n-k}
\end{aligned}
$$

\section{A CASE STUDY}

A Continuous Ship Unloader (CSU) is one of the leading bulk material handling machine. In a coal-fired power plant, this machine is used to transport coal from the vessel to the pulverized boiler through a series of belt conveyors. It has been reported that the CSU machine in one of the power plants in Malaysia has a frequent harmonic failure. The repetitive incidents lead to catastrophic failure, which harms the electrical devices. Besides having a vast replacement cost for the faulty parts, it is also affecting the plant availability which concerned the management team. Even worse, it could pose a potential hazard, to the personnel working in the area if it is happening again in the future. A thorough Power Quality (PQ) assessment within the electrical distribution system has been assessed, but the results have not given any indication of internal disturbance or fault. Table II shows the assessment result for the CSU machine.

TABLE II

\begin{tabular}{|c|c|c|c|c|}
\hline Parameters & & Min & Max & Avg \\
\hline \multirow[t]{4}{*}{ Voltage } & Vab & $385.9 \mathrm{~V}$ & $424.0 \mathrm{~V}$ & $411.2 \mathrm{~V}$ \\
\hline & Vbc & $216.8 \mathrm{~V}$ & $423.8 \mathrm{~V}$ & $382.4 \mathrm{~V}$ \\
\hline & Vca & $241.8 \mathrm{~V}$ & $424.1 \mathrm{~V}$ & $381.5 \mathrm{~V}$ \\
\hline & Ia & $32 \mathrm{~A}$ & $1718 \mathrm{~A}$ & $525.8 \mathrm{~A}$ \\
\hline \multirow[t]{2}{*}{ Current } & $\mathrm{Ib}$ & $46 \mathrm{~A}$ & $1870 \mathrm{~A}$ & $639.9 \mathrm{~A}$ \\
\hline & Ic & $30 \mathrm{~A}$ & $1568 \mathrm{~A}$ & $217.6 \mathrm{~A}$ \\
\hline Voltage Unbalance & $\% \mathrm{VU}$ & $41.6 \%$ & $0.1 \%$ & $2.6 \%$ \\
\hline \multirow[t]{2}{*}{ Current Unbalance } & $\% \mathrm{CU}$ & $16.7 \%$ & $8.8 \%$ & $52.8 \%$ \\
\hline & THDv ab & $0.4 \%$ & $1.6 \%$ & $1.0 \%$ \\
\hline \multirow[t]{3}{*}{ THD Voltage } & THDv bc & $0.3 \%$ & $1.5 \%$ & $1.0 \%$ \\
\hline & THDv ac & $0.5 \%$ & $1.9 \%$ & $1.1 \%$ \\
\hline & THDi ab & $1.9 \%$ & $20.8 \%$ & $4.5 \%$ \\
\hline \multirow{3}{*}{ THD Current } & THDi bc & $1.8 \%$ & $25.8 \%$ & $3.7 \%$ \\
\hline & THDi ac & $1.9 \%$ & $82.2 \%$ & $12.0 \%$ \\
\hline & TDDi ab & $0.1 \%$ & $2.6 \%$ & $1.3 \%$ \\
\hline \multirow[t]{2}{*}{ TDD Current } & TDDi bc & $0.1 \%$ & $1.8 \%$ & $1.2 \%$ \\
\hline & TDDi ac & $0.1 \%$ & $2.2 \%$ & $0.8 \%$ \\
\hline Frequency & $\mathrm{f}(\mathrm{Hz})$ & 49.77 & 50.13 & 49.98 \\
\hline
\end{tabular}

Assessment Results FOR CSU MACHINE

To mitigate the problem, an effort to analyse both internal and environment parameter that has a significant impact on the system is highly desirable. The authors are keen to embrace the event modeller techniques, to evaluate the significant correlation between the output and input which could cause harmonic failures in the system.

\section{A. CSU Real-time Simulation Application}

A real-time simulation which incorporates the CSU machine parameters and the event modeller algorithm was developed using National Instruments LabVIEW. For simulation purposes, the application was design based on the following:

1) 8 Event Data

2) 8 Triggered Data

3) $5 \%$ Threshold level

4) 15 minutes sampling time 

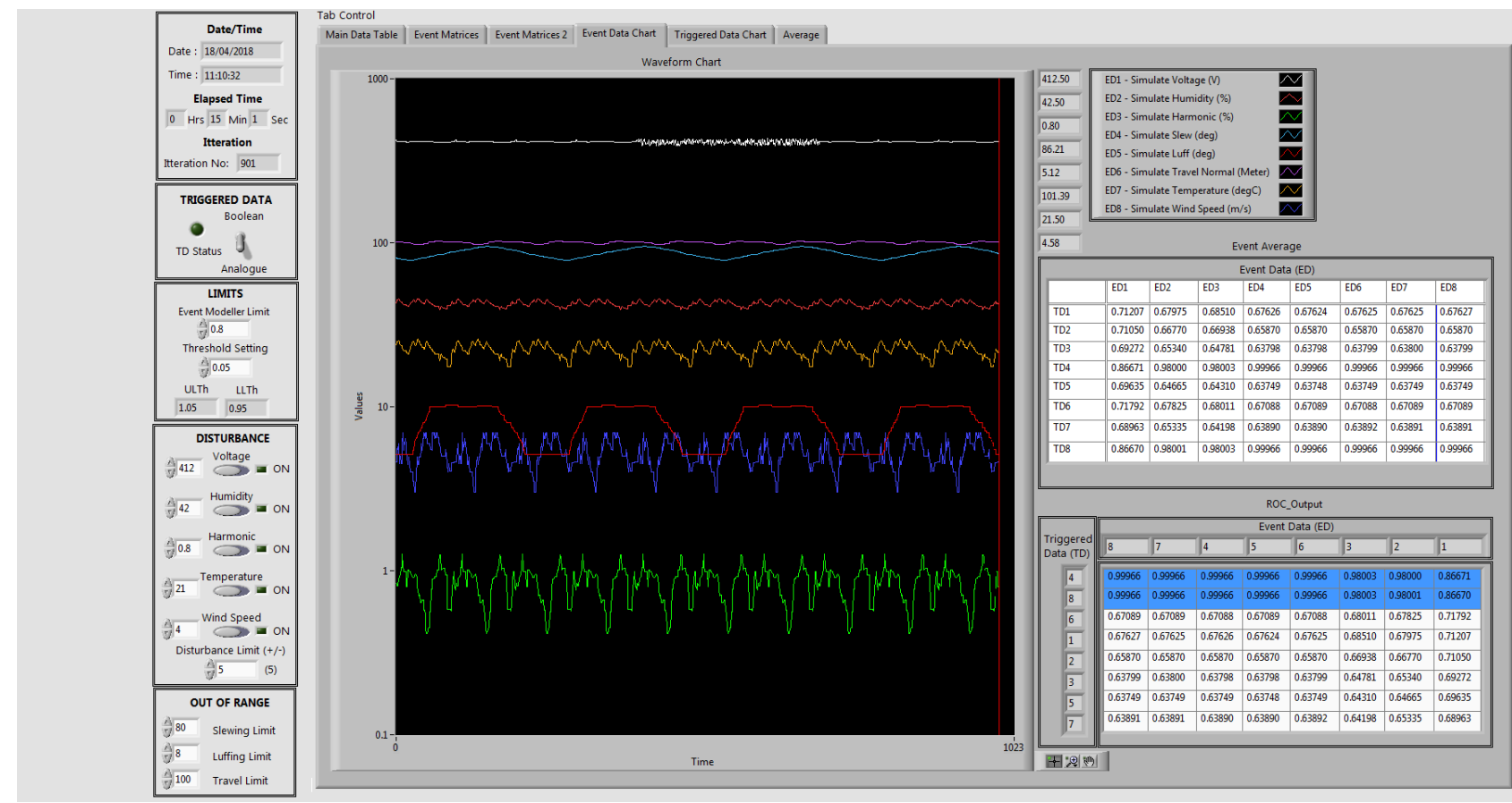

Fig. 3. Snapshot of the CSU Real-time Data Simulation

Event Data (ED) is defined as a series of data that represent the state of the system at a given time [28]. In this simulation, the ED consists of simulated voltage, humidity, harmonics, machine positioning (slewing, luffing and travelling), temperature and wind speed. The simulation data is based on the actual operation and environment data of the CSU machine events by considering the assessment result in Table II and the location of the power plant which located in Malaysia. The plant is located near to the seaside which is hot and humid throughout the year and may tend to have strong winds. On the other hand, the machine movements are simulated based on the 3-axis movement which includes slew (x-axis), luffing (y-axis) and travel (z-axis). Table III summarised the Event Data Simulation Parameters.

In Discrete Event System, any input variable whose value transition register as an event is defined as a Trigger Data (TD) [28]. In this simulation, the TD consists of machine status (slewing, luffing, travel and bucket) and motor run feedback (slewing, HPP, travel and bucket). Table IV shows the Triggered Data Simulation Parameters setting. For comparison purposes, two types of TD are presented here known as Boolean (Bool) TD and Analogue (Analog) TD. The Bool TD register the original TD signal from the source while the Analog TD compare the current TD signal with the previous TD signal using X-NOR logic. These two types of TD are important to determine which method is more effective when dealing with actual real-time data. For analogue TD, the value will be 1 when both of the TD sequences are the same value. Otherwise, it is 0 . The application is designed with a toggle switch to swap between the Bool TD and Analog TD.

Meanwhile, the threshold level could be adjusted based on the expert point of view. In this simulation, the threshold
TABLE III

Event Data Simulation Parameters

\begin{tabular}{clcccc}
\hline \hline \multirow{2}{*}{ No } & \multicolumn{1}{c}{ Description } & \multicolumn{2}{c}{ Normal } & \multicolumn{2}{c}{ Disturbance } \\
\cline { 3 - 6 } & & Min & Max & Min & Max \\
\hline ED1 & Simulated Voltage (V) & 409 & 415 & 392 & 432 \\
ED2 & Simulated Humidity (\%) & 39 & 45 & 32 & 52 \\
ED3 & Simulated Harmonic (\%) & 0.4 & 1.2 & 0 & 3 \\
ED4 & Simulated Slew (angle) & 78 & 95 & N/A & N/A \\
ED5 & Simulated Luffing (angle) & 5 & 10 & N/A & N/A \\
ED6 & Simulated Travel (meter) & 98 & 101 & N/A & N/A \\
ED7 & Simulated Temperature (degC) & 16 & 26 & 16 & 32 \\
ED8 & Simulated Wind Speed (m/s) & 0 & 8 & 4 & 16 \\
\hline \hline
\end{tabular}

TABLE IV

Triggered Data Simulation Parameters

\begin{tabular}{clcccc}
\hline \hline \multirow{2}{*}{ No } & \multicolumn{1}{c}{ Description } & \multicolumn{2}{c}{ Normal } & \multicolumn{2}{c}{ Disturbance } \\
\cline { 3 - 6 } & & Min & Max & Min & Max \\
\hline TD1 & Busy Slewing Operation & 0 & 1 & N/A & N/A \\
TD2 & Busy Luffing Operation & 0 & 1 & N/A & N/A \\
TD3 & Busy Travel Operation & 0 & 1 & N/A & N/A \\
TD4 & Busy Bucket Elevator Operation & 0 & 1 & N/A & N/A \\
TD5 & Slewing Motor Run Bit & 0 & 1 & N/A & N/A \\
TD6 & Hydraulic Motor Run Bit & 0 & 1 & N/A & N/A \\
TD7 & Travel Motor Run Bit & 0 & 1 & N/A & N/A \\
TD8 & Bucket Motor Run Bit & 0 & 1 & N/A & N/A \\
\hline \hline
\end{tabular}

level is set at $5 \%(0.05)$; thus the Upper Limit and Lower Limit will automatically set to 1.05 and 0.95 consecutively. The limits will be multiplied to the individual data computationally, to calculate the changes of the current data to the previous data and reflects to the algorithm for weight score using X-NOR logic.The event modeller limit is the desired weight limits which also could be adjusted based on the expert point of view. In this simulation, the event 
TABLE V

CSU Real-time Data Simulation Results Based on Disturbance

\begin{tabular}{|c|c|c|c|c|c|c|c|c|c|c|}
\hline \multirow{2}{*}{ Description } & \multicolumn{2}{|c|}{ Voltage } & \multicolumn{2}{|c|}{ Humidity } & \multicolumn{2}{|c|}{ Harmonic } & \multicolumn{2}{|c|}{ Temperature } & \multicolumn{2}{|c|}{ Wind Speed } \\
\hline & Bool & Analog & Bool & Analog & Bool & Analog & Bool & Analog & Bool & Analog \\
\hline Overall Maximum Weight & 0.83877 & 0.99966 & 0.83646 & 0.99965 & 0.83702 & 0.99975 & 0.83604 & 1.00000 & 0.83408 & 1.00000 \\
\hline Overall Minimum Weight & 0.50000 & 0.62878 & 0.50000 & 0.63719 & 0.50000 & 0.63609 & 0.50000 & 0.63128 & 0.50000 & 0.63252 \\
\hline k-Disturbance Weight - Pre & 0.83450 & 0.98900 & 0.82877 & 0.97900 & 0.81817 & 0.97919 & 0.83063 & 1.00000 & 0.84240 & 1.00000 \\
\hline k-Disturbance Weight - During & 0.76154 & 0.80654 & 0.77812 & 0.83042 & 0.76374 & 0.81377 & 0.79774 & 0.89764 & 0.78482 & 0.81037 \\
\hline k-Disturbance Weight - Post & 0.78132 & 0.86671 & 0.80119 & 0.87890 & 0.78976 & 0.86503 & 0.80875 & 0.93103 & 0.80350 & 0.86546 \\
\hline
\end{tabular}

modeller limit is set at (0.8). The weights who score above the Event Modeller Limit is shaded in the ROC Output table, indicates a significant correlation between the ED and the TD. The sequence of TD's and ED's is updated every second and are re-arrangeable according to the weighted score. The weighted score can take a value of 0.5 and 1 . The value is 1 when both or none of the input/output are triggered. Otherwise, it is 0.5 . The weighted score is then averaged based on the number of iteration. Having the weight score in real-time, system engineers could easily notify the management team if there is any disturbance occurs in the system state by looking at the sequence and the weights. For trending purposes, a waveform chart is presented to improve the visibility of the data. Figure 3 is the snapshot of the CSU real-time data simulation.

\section{B. Disturbance Signal}

The purpose of this simulation is to test the applicability of the Event Modeller algorithm in handling real-time data, thus observing the reaction of the system to the abnormal events. To ensure the system is sensitive to this abnormality, a k-Disturbance signal is introduced to the system. In this simulation, the data is simulated in 3 stages known as predisturbance, k-disturbance and post-disturbance. The predisturbance refers to the warm-up stage which represents the machine normal steady state. The k-disturbance refers to the fluctuation of the k-event Data, in such generating disturbance to the system. The post-disturbance refers to the reaction of the abnormal system back to normal steady state. A 5 minutes time interval is selected for each stage, which accumulates to 15 minutes of sampling time.

In this simulation, 5 ED's signal, which represent the internal and environment parameter has been chosen to be disturbed. This includes voltage, humidity, harmonic, temperature and wind speed. The signal data are simulated based on random fluctuation with 5\% disturbance limit from normal operation data capability. Table III shows the disturbance ranges for the ED's signal.

\section{Simulation Results}

Table $\mathrm{V}$ above compares the results obtained from the simulation of the CSU machine against the k-disturbance signal. What stands out in the table is that the value of the weight has changed drastically between the pre-disturbance and during disturbance for all case. For e.g., when the kdisturbance is applied to the machine voltage using Bool TD, the weight value drop from 0.83450 to 0.76154 . However, when the k-disturbance is removed, the weight value rose to 0.78132 . These indicate that the system parameters are sensitive to the disturbance signal introduced in the system.

As shown in Figure 3, the ROC Output box on the right bottom corner represents the causal-effect relationship between the simulated triggered data and simulated event data. Closer inspection of the matrices table shows that the shaded area represents the event modeller limit for the voltage disturbance data. These determine which main input data have a genuine impact on the output data. In this example, TD4 and TD8 are the main parameters for the input parameter. Further observation shows that the highest weight is 0.99966, which represent the ED8, ED7, ED4, ED5 and ED6. It follows by ED3 and ED2, which score second highest of 0.98003. It is interesting to note that the lowest weight is represented by the affected system output parameter, ED1 with the weight score of 0.86670 . Taken together, these results suggest that there is an association between TD4 with ED1 and TD8 with ED1. Thus, formulating a new unknown relationship and further classify using learning techniques. Alternatively, when the simulation is repeated with other k-disturbance, such as temperature, a significant result is expected with the temperature parameter will score the lowest weight. These results confirm the association between the triggered data with the k-disturbance event data and form a new relationship.

In accordance with the present results, previous studies have demonstrated that the power quality disturbance could be automatically classified using internal parameters within the signal processing system state. The results of this study indicate that a new parameter, outside of the signal processing system state, such as environment parameter, have potential influence in solving the PQ disturbance problem in the future.

\section{CONCLUSIONS}

This paper presented a real-time data simulation for Continuous Ship Unloader machine to suggest the possible root cause of the harmonic failures. There is a gap in the existing PQ Disturbance which does not consider external or environment parameters in the analysis. The proposed Event Modeller technique which is low in computational effort has the potential to fill up this gap while having an advantage over the conventional method which struggles with accuracy. The purpose of this simulation is to test the applicability of the Event Modeller algorithm in real-time handling, thus observing the reaction of the system to abnormal events. 
The technique is capable of grouping the high correlation system parameters together, to form an input-output relationship, which is not limited to internal parameters only. A real-time simulation which incorporates the CSU machine parameters and the event modeller algorithm was developed using National Instruments LabVIEW. 5 out of 8 event data has been simulated with a disturbance signal to observe the reaction to the system state. Results have shown that the parameters weight and sequence had been affected when the disturbance signal is introduced, and it recovers when the disturbance signal is removed. This parameter will help system engineers to build on cause-effect relationships of events that potentially link to the repetitive harmonic failures. For future work, the system will be integrated with the actual plant data to help system engineer solve the harmonic issues.

\section{ACKNOWLEDGEMENT}

For considerable improvements of the paper, we would like to thank the Editor, as well as anonymous referees. This work would not have been possible without the financial support of our government sponsorship, MARA.

\section{REFERENCES}

[1] S. Tavakoli, A. Mousavi, and S. Poslad, "Input variable selection in time-critical knowledge integration applications: A review, analysis, and recommendation paper," Advanced Engineering Informatics, vol. 27, no. 4, pp. 519-536, 2013.

[2] M. Danishvar, A. Mousavi, and P. Broomhead, "Eventic: A real-time unbiased event-based learning technique for complex systems," IEEE Transactions on Systems, Man, and Cybernetics: Systems, 2018.

[3] M. Panteli and P. Mancarella, "Influence of extreme weather and climate change on the resilience of power systems: Impacts and possible mitigation strategies," Electric Power Systems Research, vol. 127, pp. 259-270, 2015.

[4] M. K. Saini and R. Kapoor, "Classification of power quality events-a review," International Journal of Electrical Power \& Energy Systems, vol. 43, no. 1, pp. 11-19, 2012.

[5] M. Danishvar, A. Mousavi, and P. Sousa, "Eventclustering for improved real time input variable selection and data modelling," in Control Applications (CCA), 2014 IEEE Conference on. IEEE, 2014, pp. 1801-1806.

[6] W. R. A. Ibrahim and M. M. Morcos, "Artificial intelligence and advanced mathematical tools for power quality applications: a survey," IEEE Transactions on Power Delivery, vol. 17, no. 2, pp. 668-673, Apr 2002.

[7] M. Morcos and W. A. Ibrahim, "Electric power quality and artificial intellignece: Overview and applicability," IEEE Power engineering review, vol. 19 , no. 6, pp. 5-10, 1999.

[8] D. Saxena, K. Verma, and S. Singh, "Power quality event classification: an overview and key issues," International Journal of Engineering, Science and Technology, vol. 2, no. 3, pp. 186-199, 2010.

[9] D. C. Robertson, O. I. Camps, J. S. Mayer, and W. B. Gish, "Wavelets and electromagnetic power system transients," IEEE Transactions on Power Delivery, vol. 11, no. 2, pp. 1050-1058, 1996.

[10] S. Santoso, E. J. Powers, W. M. Grady, and P. Hofmann, "Power quality assessment via wavelet transform analysis," IEEE transactions on Power Delivery, vol. 11, no. 2, pp. 924-930, 1996.

[11] J. Barros and R. I. Diego, "Analysis of harmonics in power systems using the wavelet-packet transform," IEEE Transactions on Instrumentation and Measurement, vol. 57, no. 1, pp. 63-69, 2008.

[12] S. Shukla, S. Mishra, and B. Singh, "Empirical-mode decomposition with hilbert transform for power-quality assessment," IEEE transactions on power delivery, vol. 24, no. 4, pp. 2159-2165, 2009.

[13] R. G. Stockwell, "A basis for efficient representation of the stransform," Digital Signal Processing, vol. 17, no. 1, pp. 371-393, 2007.

[14] S.-H. Cho, G. Jang, and S.-H. Kwon, "Time-frequency analysis of power-quality disturbances via the gabor-wigner transform," IEEE transactions on power delivery, vol. 25, no. 1, pp. 494-499, 2010.
[15] C. Norman, J. Y. Chan, W. H. Lau, and L. L. Lai, "Hybrid wavelet and hilbert transform with frequency-shifting decomposition for power quality analysis." IEEE Trans. Instrumentation and Measurement, vol. 61, no. 12, pp. 3225-3233, 2012.

[16] K. Manimala, K. Selvi, and R. Ahila, "Optimization techniques for improving power quality data mining using wavelet packet based support vector machine," Neurocomputing, vol. 77, no. 1, pp. 36-47, 2012.

[17] R. Ahila, V. Sadasivam, and K. Manimala, "An integrated pso for parameter determination and feature selection of elm and its application in classification of power system disturbances," Applied Soft Computing, vol. 32, pp. 23-37, 2015.

[18] B. Biswal, P. K. Dash, and S. Mishra, "A hybrid ant colony optimization technique for power signal pattern classification," Expert Systems with Applications, vol. 38, no. 5, pp. 6368-6375, 2011.

[19] C. Cortes and V. Vapnik, "Support-vector networks," Machine learning, vol. 20, no. 3, pp. 273-297, 1995.

[20] L. A. Zadeh, "Fuzzy sets," in Fuzzy Sets, Fuzzy Logic, And Fuzzy Systems: Selected Papers by Lotfi A Zadeh. World Scientific, 1996, pp. 394-432.

[21] S. Khokhar, A. M. Zin, A. Mokhtar, N. M. Ismail, and N. Zareen, "Automatic classification of power quality disturbances: a review," in Research and Development (SCOReD), 2013 IEEE Student Conference on. IEEE, 2013, pp. 427-432.

[22] O. P. Mahela, A. G. Shaik, and N. Gupta, "A critical review of detection and classification of power quality events," Renewable and Sustainable Energy Reviews, vol. 41, pp. 495-505, 2015.

[23] D. Granados-Lieberman, R. Romero-Troncoso, R. Osornio-Rios, A. Garcia-Perez, and E. Cabal-Yepez, "Techniques and methodologies for power quality analysis and disturbances classification in power systems: a review," IET Generation, Transmission \& Distribution, vol. 5, no. 4, pp. 519-529, 2011.

[24] M. McGranaghan, "Overview of power quality standards," $P Q$ Network Internet Site, http://www. pqnet. electrotek. com/pqnet, 1998.

[25] I. . W. Group et al., "Ieee recommended practices and requirements for harmonic control in electrical power systems," IEEE STD, pp. 5191992, 1992.

[26] B. IEC, "61000-3-4," Electromagnetic compatibility (EMC)-part, pp. 3-4, 1998.

[27] P. Muchiri and L. Pintelon, "Performance measurement using overall equipment effectiveness (oee): literature review and practical application discussion," International journal of production research, vol. 46, no. 13 , pp. $3517-3535,2008$.

[28] S. Tavakoli, A. Mousavi, and P. Broomhead, "Event tracking for realtime unaware sensitivity analysis (eventtracker)," IEEE Transactions on Knowledge and Data Engineering, vol. 25, no. 2, pp. 348-359, 2013.

[29] S. Tavakoli, A. Mousavi, and A. Komashie, "A generic framework for real-time discrete event simulation (des) modelling," in Proceedings of the 40th Conference on Winter Simulation. Winter Simulation Conference, 2008, pp. 1931-1938.

[30] J. R. King, "Machine-component grouping in production flow analysis: an approach using a rank order clustering algorithm," International Journal of Production Research, vol. 18, no. 2, pp. 213-232, 1980. 